Geometry 83 Topology Monographs

Volume 7: Proceedings of the Casson Fest

Pages 181-203

\title{
On Heegaard Floer homology and Seifert fibered surgeries
}

\author{
Peter Ozsváth \\ ZOLTÁN SZABÓ
}

\begin{abstract}
We explore certain restrictions on knots in the three-sphere which admit non-trivial Seifert fibered surgeries. These restrictions stem from the Heegaard Floer homology for Seifert fibered spaces, and hence they have consequences for both the Alexander polynomial of such knots, and also their knot Floer homology. In particular, we show that certain polynomials are never the Alexander polynomials of knots which admit homology three-sphere Seifert fibered surgeries. The knot Floer homology restrictions, on the other hand, apply also in cases where the Alexander polynomial gives no information, such as the Kinoshita-Terasaka knots.
\end{abstract}

AMS Classification 57R58; 57M25

Keywords Floer homology, Seifert fibered surgeries

\section{Introduction}

It is an interesting open question to characterize knots $K$ in the three-sphere with the property that some (non-trivial) surgery on $S^{3}$ along $K$ is a Seifert fibered space. This question has received considerable attention recently, cf 2. 9, 3. The aim of the present article is to present some obstructions for a knot $K$ to admitting such surgeries. These obstructions in turn come from the Heegaard Floer homology of [19] and the related knot invariant defined in [21] and [28].

\subsection{Seifert fibered surgeries and the Alexander polynomial}

For simplicity, throughout most of this paper we consider the case of homology sphere surgeries, although other surgeries are accessible to Floer homology as well (compare also [13, Subsection 8.1]). Specifically, given a rational number 
$r$ and a knot $K \subset S^{3}$, let $S_{r}^{3}(K)$ denote the three-manifold obtained as Dehn surgery on $S^{3}$ along $K$. Writing $r=p / q$ as a fraction in its lowest terms, $H_{1}\left(S_{p / q}^{3}(K) ; \mathbb{Z}\right) \cong \mathbb{Z} / p \mathbb{Z}$. In particular, if $S_{r}^{3}(K)$ is an integer homology threesphere, then $r$ has the form $1 / q$ for a non-zero integer $q$.

We give a certain obstruction to Seifert fibered surgeries. The strongest form of this result should be stated in terms of the Heegaard Floer homology of the zero-surgery of $S^{3}$ along $K, H F^{+}\left(S_{0}^{3}(K)\right)$ cf Theorem 3.4 and Proposition 3.5 below. However, for the purposes of this introduction, we prefer to state a weaker form in terms of its Euler characteristic, which can be expressed purely in terms of the Alexander polynomial. (This result is proved in Section 3])

Theorem 1.1 Let $K \subset S^{3}$ be a knot in the three-sphere. Write its symmetrized Alexander polynomial as

$$
\Delta_{K}(T)=a_{0}+\sum_{i>0} a_{i}\left(T^{i}+T^{-i}\right)
$$

and let

$$
t_{i}(K)=\sum_{j=1}^{\infty} j a_{|i|+j}
$$

denote the torsion coefficients of the knot. Then, if there is an integer $q \neq 0$ for which $S_{1 / q}^{3}(K)$ is Seifert fibered, all the non-zero integers $t_{i}(K)$ have the same sign.

For example, for 34 of the 54 non-alternating knots with fewer than eleven crossings, there are both positive and negative torsion coefficients. It follows from Theorem 1.1 that these knots admit no Seifert fibered $1 / q$-surgeries. (Of course, there are other known techniques for ruling out Seifert fibered surgeries for sufficiently small knots, cf [30.)

This result rules out many alternating knots, as well. In particular, there is an interesting family which we describe below. But first, recall that the sign of the $t_{i}(K)$ is governed by the following result, proved in [23]:

Theorem 1.2 (Corollary 1.6 of [23]) Let $K$ be an alternating knot, and let

$$
\delta(m, i)=\max \left(0,\left\lceil\frac{|m|-2|i|}{4}\right\rceil\right)
$$

(note that this is the $i^{\text {th }}$ torsion coefficient of the $(2,2 m+1)$ torus knot). Then,

$$
(-1)^{i+\frac{\sigma}{2}}\left(t_{i}(K)-\delta(\sigma, i)\right) \leq 0,
$$

where here $\sigma$ denotes the signature of $K$. 
We now have the following result:

Corollary 1.3 If $K$ is an alternating knot with genus $g$ and whose signature $\sigma$ satisfies

$$
g+\frac{\sigma}{2} \equiv 1 \quad(\bmod 2) \quad \text { and } \quad \sigma \neq 0
$$

then there is no non-zero integer $q \neq 0$ for which $S_{1 / q}^{3}(K)$ is Seifert fibered.

Proof It is a classical result that the genus $g$ of an alternating knot agrees with the degree of its Alexander polynomial (cf [1, 15]). In particular, $t_{g-1}(K) \neq 0$.

Recall that in general $|\sigma| \leq 2 g$, while our hypothesis on the parity of $\sigma / 2$ forces this inequality to be strict, and hence $\delta(\sigma, g-1)=0$. Theorem 1.2 together with the hypothesis on the parity of $\sigma / 2$ again, gives

$$
t_{g-1}(K)<0 \text {. }
$$

On the other hand, letting $i=\frac{|\sigma|}{2}-1$, we have that $\delta(\sigma, i)=1$, and hence another application of Theorem 1.2 gives

$$
1 \leq t_{i}
$$

Together, inequalities (2) and (3), combined with Theorem 1.1, give the claimed result.

To elucidate the hypotheses of the above corollary, note that the figure-eight knot satisfies the parity hypothesis, but $\sigma=0$. However, +1 -surgery on this knot gives the Brieskorn sphere $\Sigma(2,3,7)$. Moreover, for each integer $n \geq 1$, the $(2,2 n+1)$ torus knot has non-zero signature $(2 n)$, but $\sigma / 2=g$. Of course, +1 surgery on any of these knots is Seifert fibered.

The sign of the torsion coefficients as in Theorem 1.1 is dictated by the orientation on the Seifert fibered space. Specifically, note that for any integral homology Seifert fibered space $Y$ different from $S^{3}$, Casson's invariant $\lambda(Y)$ is non-zero (cf [4, 16, 7]). Moreover, Casson's surgery formula shows that

$$
\lambda\left(S_{1 / q}^{3}(K)\right)=q \cdot \sum_{i} t_{i}(K) .
$$

Putting these two together, we see that the signs of the non-vanishing $t_{i}$ are determined by Casson's invariant of $Y$. We say that $Y$ a Seifert fibered integral homology three-sphere has a positive Seifert orientation if its Casson's invariant is positive (ie has the same sign as that of the Brieskorn sphere $-\Sigma(2,3,5)$ ). In Section 3 we give a more direct description of this sign. 


\subsection{Knot Floer homology}

We have also a Seifert fibered surgery obstruction, which can be stated in terms of the "knot Floer homology". This invariant is a refinement of Heegaard Floer homology of the three-manifold $Y$, in the presence of an oriented knot $K$ in $Y,[21,28$. We will say little about this invariant beyond its formal properties. If $K$ is a null-homologous knot in a three-manifold $Y$, then there is an induced filtration of the Heegaard Floer complex, and the homology of the associated graded object is this knot Floer homology.

For knots $K$ in the three-sphere, the invariants $\widehat{H F K}$ take the form of graded Abelian groups indexed by integers $i$. According to a "skein exact sequence" which these groups satisfy, it follows that if $\Delta_{K}(T)$ denotes the symmetrized Alexander polynomial of $K$, then

$$
\Delta_{K}(T)=\sum_{i \in \mathbb{Z}} \chi(\widehat{H F K}(K, i)) \cdot T^{i} \in \mathbb{Z}\left[T, T^{-1}\right]
$$

(see [21, Proposition 4.2]).

Moreover, according to [26, Theorem 1.2], the knot Floer homology determines the genus of the knot:

$$
g(K)=\max \{i \mid \widehat{H F K}(K, i) \neq 0\} .
$$

Knot Floer homology is difficult to calculate in general. However, there are some families of knots for which the answer is known. For example, if $K$ is an alternating knot, then $\widehat{H F K}(K, i) \cong \mathbb{Z}^{\left|a_{i}\right|}$ is supported in dimension $i-\frac{\sigma}{2}$, where $\sigma$ denotes the signature of $K$ (cf [23. Theorem 1.4], see also [27] for the two-bridge case). Here, of course, $a_{i}$ is the $T^{i}$ coefficient of the Alexander polynomial of $K$.

\subsection{Seifert fibered surgeries and knot Floer homology}

Theorem 3.4 together with some additional properties of the knot Floer homology which we review in Section 4, give strong restrictions on $\widehat{H F K}(K)$ for knots which admit Seifert fibered surgeries. This restriction leads to the following:

Theorem 1.4 Let $K \subset S^{3}$ be a knot with genus $g$. If there is an integer $q>0$ so that $S_{1 / q}^{3}(K)$ is a positively oriented Seifert fibered space, then $\widehat{H F K}\left(S^{3}, K, g\right)$ is trivial in odd degrees (and non-trivial in even degrees). If 
there is an integer $q>0$ so that $S_{1 / q}^{3}(K)$ is a negatively oriented Seifert fibered space and $g>1$, then $\widehat{H F K}\left(S^{3}, K, g\right)$ is trivial in even degrees (and non-trivial in odd degrees).

This has the following corollary (compare also [13]):

Corollary 1.5 If $\operatorname{deg} \Delta_{K}<g(K)$, then for integers $q \geq 0, S_{1 / q}^{3}(K)$ is never a positively oriented Seifert fibered space. Indeed, if in addition, $g(K)>1$ then no $1 / q$ surgery along $K$ is Seifert fibered.

Proof Note that $S_{1 / q}^{3}(K)=-S_{-1 / q}^{3}(r(K))$, where $r(K)$ denotes the reflection of $K$. Thus, the corollary follows immediately from the Euler characteristic relation (equation (4)), together with Theorem 1.4.

As an illustration, we consider the family of Kinoshita-Terasaka knots $K T_{r, n}$ (see [1]) with $|r| \geq 2$ and $n \neq 0$ (to avoid the unknot). These knots all have trivial Alexander polynomial. However, Gabai has shown [8] that $K T_{r, n}$ has genus $|r|$ (note that one could deduce this alternatively from the calculation of $\widehat{H F K}\left(K_{r, n}, r\right)$ from [24]). Thus, Corollary 1.5 applies: none of these knots admits surgeries which are integral homology Seifert fibered spaces. See [24] for other families of knots with these properties.

In this paper, we have dealt with restrictions on Seifert fibered surgeries which are obtained with the use of Heegaard Floer homology. Further results on Seifert fibered surgeries using monopole Floer homology can be found in 13 , Subsection 8.1].

\subsection{Surgeries giving $\Sigma(2,3,5)$ and $\Sigma(2,3,7)$}

It is conjectured that if $K$ is a knot in $S^{3}$ which admits a surgery which is $\Sigma(2,3,5)$, then $K$ is the trefoil (cf remarks following [12, Problem 3.6(D)], see also [31]), and if $K$ is a knot in $S^{3}$ which admits a surgery which is $\Sigma(2,3,7)$, then $K$ is the figure-eight or the trefoil. We use the surgery long exact sequence to provide some evidence for this conjecture in the following form:

Theorem 1.6 Let $K$ be a knot in $S^{3}$ with the property that $S_{r}^{3}(K) \cong$ $\Sigma(2,3,5)$ for some $r \in \mathbb{Q}$, then $r=-1$, and the knot Floer homology of $K$ agrees with that of the left-handed trefoil. In particular, the Seifert genus of $K$ is one and its Alexander polynomial is $T^{-1}-1+T$. 
Theorem 1.7 Let $K$ be a knot in $S^{3}$ with the property that $S_{r}^{3}(K) \cong$ $\Sigma(2,3,7)$ (as an oriented manifold) then there are two cases. Either $r=-1$, in which case $K$ has genus one and its Alexander polynomial agrees with that of the trefoil. In the case where $r=+1, K$ has genus one and its Alexander polynomial is $-T^{-1}+3-T$.

\subsection{Acknowledgements}

The authors would like to thank Cameron Gordon for helpful discussions on these questions. The first author was partially supported by NSF grant numbers DMS-0234311, DMS-0111298, and FRG-0244663, and the second author was partially supported by NSF grant numbers DMS-0107792 and FRG-0244663, and a Packard Fellowship.

\section{A brief review of Heegaard Floer homology}

Very briefly, the Heegaard Floer homology is a homology theory associated to a closed, oriented three-manifold $Y$. For simplicity, we start with the case where $Y$ is an integral homology three-sphere. To define Heegaard Floer homology, we start with a suitably generic Heegaard diagram $(\Sigma, \boldsymbol{\alpha}, \boldsymbol{\beta}, z)$, where here $\Sigma$ is an oriented Riemann surface, and $\boldsymbol{\alpha}=\left\{\alpha_{1}, \ldots, \alpha_{g}\right\}$ and $\boldsymbol{\beta}=\left\{\beta_{1}, \ldots \beta_{g}\right\}$ are $g$-tuples of (pairwise disjoint, embedded) attaching circles for the two handlebodies, and $z$ is a point which does not lie on any of the attaching circles. We now consider the $g$-fold symmetric product $\operatorname{Sym}^{g}(\Sigma)$, ie unordered $g$-tuples of points (counted with multiplicity) on $\Sigma$, together with the pair of embedded tori

$$
\mathbb{T}_{\alpha}=\alpha_{1} \times \ldots \times \alpha_{g} \quad \text { and } \quad \mathbb{T}_{\beta}=\beta_{1} \times \ldots \times \beta_{g} .
$$

Loosely speaking, Heegaard Floer homology measures an obstruction to pulling apart these two tori. More precisely, we fix a complex structure on $\Sigma$, which in turn induces a complex structure on its $g$-fold symmetric product (with respect to which the two tori are totally real), and we consider the chain complex $C F^{\infty}(Y)$ generated by $[\mathbf{x}, i] \in\left(\mathbb{T}_{\alpha} \cap \mathbb{T}_{\beta}\right) \times \mathbb{Z}$, and whose differential counts holomorphic Whitney disks. That is, fix intersection points $\mathbf{x}, \mathbf{y} \in \mathbb{T}_{\alpha} \cap \mathbb{T}_{\beta}$. A Whitney disk $u$ connecting $\mathbf{x}$ to $\mathbf{y}$ is a map

$$
u:\{\mathbf{z} \in \mathbb{C}|| z \mid \leq 1\} \longrightarrow \operatorname{Sym}^{g}(\Sigma)
$$


satisfying the boundary conditions

$$
\begin{aligned}
u\{\zeta \mid \operatorname{Re}(\zeta) \geq 0 \text { and }|\zeta|=1\} \subset \mathbb{T}_{\alpha}, & u\{\zeta \mid \operatorname{Re}(\zeta) \leq 0 \text { and }|\zeta|=1\} \subset \mathbb{T}_{\beta}, \\
u(-\sqrt{-1})=\mathbf{x}, & u(\sqrt{-1})=\mathbf{y} .
\end{aligned}
$$

The space of homotopy classes of Whitney disks connecting $\mathbf{x}$ to $\mathbf{y}$ is denoted $\pi_{2}(\mathbf{x}, \mathbf{y})$. For a fixed Whitney disk $u$, let $n_{z}(u)$ denote the algebraic intersection number of $u$ with the submanifold $\{z\} \times \operatorname{Sym}^{g-1}(\Sigma) \subset \operatorname{Sym}^{g}(\Sigma)$. Note that $n_{z}(u)$ depends only on the homotopy class $\phi$ of $u$.

With these preliminaries in place, we can define a map

$$
\partial[\mathbf{x}, i]=\sum_{\mathbf{y} \in \mathbb{T}_{\alpha} \cap \mathbb{T}_{\beta}} \sum_{\phi \in \pi_{2}(\mathbf{x}, \mathbf{y}) \mid \mu(\phi)=1} c(\phi) \cdot\left[\mathbf{y}, i-n_{z}(\phi)\right],
$$

where here $\mu(\phi)$ denotes the expected dimension of the moduli space of pseudoholomorphic representatives for the homotopy class $\phi$, and $c(\phi)$ denotes an appropriate signed count of points in this moduli space, modulo the natural automorphism group. Here the term "pseudo-holomorphic" means a sufficiently generic perturbation of the notion of holomorphic disks. By adapting arguments from Lagrangian Floer homology (which in turn rest on Gromov's compactness theorem [10]), one can show that $\partial^{2}=0$. It is simplest to think of $c(\phi)$ as an element of a field (rather than working over $\mathbb{Z}$ ), which we fix now to be the field $\mathbb{Q}$ of rational numbers.

In fact, since $\{z\} \times \operatorname{Sym}^{g-1}(\Sigma)$ is a subvariety of $\operatorname{Sym}^{g}(\Sigma)$ which is disjoint from $\mathbb{T}_{\alpha}$ and $\mathbb{T}_{\beta}$, it follows that if a given homotopy class $\phi$ has holomorphic representatives, then $n_{z}(\phi) \geq 0$. In particular, it follows that the subset of $C F^{-}(Y) \subset C F^{\infty}(Y)$ generated by pairs $[\mathbf{x}, i]$ with $i<0$ is actually a subcomplex. Indeed, we have a short exact sequence of complexes

$$
0 \longrightarrow C F^{-}(Y) \longrightarrow C F^{\infty}(Y) \stackrel{\pi}{\longrightarrow} C F^{+}(Y) \longrightarrow 0,
$$

where here $C F^{+}(Y)$ is defined to make the sequence exact; it is generated by pairs $[\mathbf{x}, i]$ with $i \geq 0$. All three complexes are endowed with an endomorphism, defined by $U[\mathbf{x}, i]=[\mathbf{x}, i-1]$, which can be used to construct a fourth variant:

$$
0 \longrightarrow \widehat{C F}(Y) \longrightarrow C F^{+}(Y) \stackrel{U}{\longrightarrow} C F^{+}(Y) \longrightarrow 0,
$$

where here $\widehat{C F}(Y)$ is, by definition, the kernel of the induced endomorphism. According to [19], the homology groups of the complexes $C F^{-}(Y), C F^{\infty}(Y)$, $C F^{+}(Y)$, and $\widehat{C F}(Y)$, denoted $H F^{-}(Y), H F^{\infty}(Y), H F^{+}(Y)$, and $\widehat{H F}(Y)$, are topological invariants of the three-manifold $Y$. In particular, they are independent of the perturbations, complex structures, and Heegaard diagrams 
which go into their definitions. In addition, the complexes can be given a canonical $\mathbb{Z} / 2 \mathbb{Z}$-grading, characterized by the properties that it is preserved by the maps in the short exact sequences (6) and (7), and also that $\chi(\widehat{H F}(Y))=1$, bearing in mind that we have assumed that $H_{1}(Y ; \mathbb{Z})=0$. Sometimes, we write $\widehat{H F}_{\text {ev }}(Y)$ (respectively $\widehat{H F}_{\text {odd }}(Y)$ ) for the subgroup of $\widehat{H F}(Y)$ generated by elements with even (respectively odd) $\mathbb{Z} / 2 \mathbb{Z}$-grading (and the corresponding notation for the other types of Heegaard Floer homology).

As a special case, consider $S^{3}$. For this manifold, $\widehat{H F}\left(S^{3}\right) \cong \mathbb{Q}, H F^{-}\left(S^{3}\right) \cong$ $\mathbb{Q}[U], H F^{\infty}\left(S^{3}\right)$

$\cong \mathbb{Q}\left[U, U^{-1}\right]$, and $H F^{+}\left(S^{3}\right) \cong \mathbb{Q}\left[U, U^{-1}\right] / U \cdot \mathbb{Q}[U]$ (thought of as a $\mathbb{Q}[U]-$ module). We denote this latter module by $\mathcal{T}^{+}$. It is interesting to note that, according to [18, Theorem 10.1],

$$
H F^{\infty}(Y) \cong \mathbb{Q}\left[U, U^{-1}\right]
$$

(supported in even parity) for any integer homology three-sphere $Y$.

In the case of a general closed, oriented three-manifold the same constructions work, except that when $b_{1}(Y)>0$, we use a restricted class of Heegaard diagram, a technical point which we will not pursue here (cf [19, Section $5]$ ). But there is some additional structure which is important to us here: the chain complex splits into summands indexed by $\operatorname{Spin}^{c}$ structures over $Y$. For example, consider the case where $Y$ is obtained by $p$-framed surgery on a knot $K \subset S^{3}$, where $p$ is an integer. In this case, there is an identification $H_{1}\left(S_{p}^{3}(K) ; \mathbb{Z}\right) \cong \mathbb{Z} / p \mathbb{Z}$. And indeed, we have a corresponding identification $\operatorname{Spin}^{c}\left(S_{p}^{3}(K)\right) \cong \mathbb{Z} / p \mathbb{Z}$. In this case, there is a splitting of all three of the Heegaard Floer homologies indexed by integers $i \in \mathbb{Z} / p \mathbb{Z}$; we write

$$
H F^{+}\left(S_{p}^{3}(K)\right) \cong \bigoplus_{i \in \mathbb{Z} / p \mathbb{Z}} H F^{+}\left(S_{p}^{3}(K), i\right)
$$

(The canonical $\mathbb{Z} / 2 \mathbb{Z}$-grading is slightly more complicated when $b_{1}(Y)>0$, but can still be defined, cf [18, Section 10.4], see also equation (13) below.)

In general, $H F^{+}(Y)$ is infinitely generated. However the quotient $H F_{\text {red }}^{+}(Y)=$ $H F^{+}(Y) / H F^{\infty}(Y)$ is finitely generated. The link between Heegaard Floer homology and the Alexander polynomial is provided by the following result, which can be seen as an analogoue of the Meng-Taubes theorem in SeibergWitten theory [14, 29].

Theorem 2.1 Let $K \subset S^{3}$ be a knot, and let $S_{0}^{3}(K)$ denote the threemanifold obtained by performing 0 -framed surgery on $S^{3}$ along $K$. Then, 
$H F^{+}\left(S_{0}^{3}(K), i\right)$ is finitely generated when $i \neq 0$, with Euler characteristic given by

$$
\chi\left(H F^{+}\left(S_{0}^{3}(K), i\right)\right)=-t_{i}(K) .
$$

In the case where $i=0$,

$$
\chi\left(H F_{\text {red }}^{+}\left(S_{0}^{3}(K), i\right)\right) \geq-t_{0}(K) .
$$

The above theorem is stated and proved in [18, Theorem 5.2] in the case where $i \neq 0$, while in the case where $i=0$, it is [18, Theorem 10.17].

A key calculational device we will use here is the following integer surgeries long exact sequence, which is a restatement of [18, Theorem 9.19]:

Theorem 2.2 Let $K \subset S^{3}$, and fix an integer $p>0$. Then, there is an long exact sequence

$$
\ldots \longrightarrow H F^{+}\left(S^{3}\right) \stackrel{a}{\longrightarrow} \bigoplus_{j \equiv i(\bmod p)} H F^{+}\left(S_{0}^{3}(K), j\right) \stackrel{b}{\longrightarrow} H F^{+}\left(S_{p}^{3}(K),[i]\right) \longrightarrow \ldots
$$

where here $a$ and $c$ preserves the canonical $\mathbb{Z} / 2 \mathbb{Z}$ degree, while $b$ reverses it.

There are many variants of this sequence. The case where $p=1$ generalizes to the case of arbitrary framed knots in an arbitrary closed, oriented threemanifold (though the statements about the $\mathbb{Z} / 2 \mathbb{Z}$ degree have to be suitably modified). (We say little about the maps on Floer homology in the exact sequence, except that they are induced from cobordisms. For more on this, see [20].)

Another variant uses fractional surgeries. The term associated to the zerosurgery is slightly different in this case. Specifically, suppose that $Y$ is a threemanifold with $H_{1}(Y ; \mathbb{Z}) \cong \mathbb{Z}$, fix a homomorphism $H^{1}(Y ; \mathbb{Z}) \longrightarrow \mathbb{Z} / p \mathbb{Z}$. Then, there is a notion of Floer homology with coefficients in $\mathbb{Q}[\mathbb{Z} / p \mathbb{Z}]$, written

$$
\underline{H F}^{+}(Y ; \mathbb{Q}[\mathbb{Z} / p \mathbb{Z}]) \cong \bigoplus_{i \in \mathbb{Z}} \underline{H F}^{+}(Y, i ; \mathbb{Q}[\mathbb{Z} / p \mathbb{Z}])
$$

In fact, this theory is obtained from a "universal" case of coefficients twisted by $\mathbb{Q}\left[H^{1}(Y ; \mathbb{Z})\right]=\mathbb{Q}[\mathbb{Z}]$ which, of course, can be thought of as the ring of Laurent polynomials with coefficients in $\mathbb{Q}$ over a formal variable $T$. In this case, the universal coefficients theorem applies, to show that

$$
\left.H F_{i}^{+}(Y ; \mathbb{Q}) \cong\left(\underline{H F}_{i}^{+}(Y ; \mathbb{Q}[\mathbb{Z}]) \otimes \mathbb{Q}\right) \oplus\left(\mathbb{Q} \star \underline{H F}_{i+1}^{+}(Y ; \mathbb{Q}[\mathbb{Z} / p \mathbb{Z}])\right)\right),
$$

where here $i$ denotes the parity $i \in \mathbb{Z} / 2 \mathbb{Z}$, tensor products are taken over the base ring $\mathbb{Q}[\mathbb{Z}]$, where here $\mathbb{Q}$ is viewed as a module over $\mathbb{Q}[\mathbb{Z}]$ with trivial 
action by $\mathbb{Z}$, and $A \star B$ denotes the torsion product over the principal ideal domain $\mathbb{Q}[\mathbb{Z}]$. More generally (ie generalizing from the case where $p=1$ ), we have

$$
\begin{aligned}
& \underline{H F}_{i}^{+}(Y ; \mathbb{Q}[\mathbb{Z} / p \mathbb{Z}]) \cong \\
& \left.\qquad(\underline{H F}+i(Y ; \mathbb{Q}[\mathbb{Z}]) \otimes \mathbb{Q}[\mathbb{Z} / p \mathbb{Z}]) \oplus\left(\mathbb{Q}[\mathbb{Z} / p \mathbb{Z}] \star \underline{H F}_{i+1}^{+}(Y ; \mathbb{Q}[\mathbb{Z} / p \mathbb{Z}])\right)\right),
\end{aligned}
$$

where here $\mathbb{Q}[\mathbb{Z} / p \mathbb{Z}]$ is a module over $\mathbb{Q}[\mathbb{Z}]$ using our given homomorphism $\mathbb{Z} \cong H^{1}(Y ; \mathbb{Z}) \longrightarrow \mathbb{Z} / p \mathbb{Z}$. Note that all of the groups $\widehat{H F}, H F^{-}, H F^{\infty}$, and $H^{+}$have their analogues with twisted coefficients, and they are related by exact sequences analogous to equations (6) and (7). In particular, we can once again form the group

$$
\underline{H F}+\mathrm{red}(Y ; \mathbb{Q}[\mathbb{Z} / p \mathbb{Z}])=\underline{H F}{ }^{+}(Y ; \mathbb{Q}[\mathbb{Z} / p \mathbb{Z}]) / \underline{H F}^{\infty}(Y ; \mathbb{Q}[\mathbb{Z} / p \mathbb{Z}]) .
$$

Again the canonical $\mathbb{Z} / 2 \mathbb{Z}$-grading gives a decomposition

$$
\underline{H F}_{\text {red }}^{+}(Y ; \mathbb{Q}[\mathbb{Z} / p \mathbb{Z}])=\underline{H F}_{\text {red,ev }}^{+}(Y ; \mathbb{Q}[\mathbb{Z} / p \mathbb{Z}]) \oplus \underline{H F}_{\text {red,odd }}^{+}(Y ; \mathbb{Q}[\mathbb{Z} / p \mathbb{Z}]) .
$$

The Euler characteristic of $\underline{H F}$ with coefficients in $\mathbb{Q}[\mathbb{Z} / p \mathbb{Z}]$ is related to the untwisted case by the relation

$$
\chi\left(\underline{H F}^{+}(Y, i, \mathbb{Q}[\mathbb{Z} / p \mathbb{Z}])\right)=p \cdot \chi\left(H F^{+}(Y, i)\right)
$$

for all $i \neq 0(\operatorname{cf}[18$, Lemma 11.1]).

We use twisted coefficients for the following variant of the surgery long exact sequence, which is a restatement of [18, Theorem 9.14]

Theorem 2.3 Let $K \subset S^{3}$ be a knot. For each integer $p>0$, there is a homomorphism $H_{1}\left(S_{0}^{3}(K) ; \mathbb{Z}\right) \longrightarrow \mathbb{Z} / p \mathbb{Z}$ for which we have a long exact sequence

$$
\ldots \longrightarrow H F^{+}\left(S^{3}\right) \stackrel{a}{\longrightarrow} \underline{H F^{+}}\left(S_{0}^{3}(K), \mathbb{Q}[\mathbb{Z} / p \mathbb{Z}]\right) \stackrel{b}{\longrightarrow} H F^{+}\left(S_{1 / p}^{3}(K)\right) \longrightarrow \ldots,
$$

where once again $a$ and $c$ preserve $\mathbb{Z} / 2 \mathbb{Z}$ degree and $b$ reverses it.

\subsection{Absolute gradings}

In the case where $Y$ is an integral homology three-sphere, the $\mathbb{Z} / 2 \mathbb{Z}$-grading can be lifted to a $\mathbb{Z}$-grading on the Floer homology of $H F^{+}(Y)$, cf [20, Section 7.1]. This provides at once a numerical invariant for integer homology three-spheres, the correction term denoted $d(Y)$, which is the minimal dimension of any homogeneous element in $H F^{+}(Y)$ coming from $H F^{\infty}(Y)$, cf [22]. 
This invariant restricts the intersection forms of smooth four-manifolds which bound $Y$, according to the following result (compare also the analogous gaugetheoretic results of Frøyshov, cf [6, [5]):

Theorem 2.4 (Corollary 9.8 of [22]) If $Y$ is an integer homology three-sphere which bounds a smooth, negative-definite four-manifold, then $d(Y) \geq 0$.

In the case where $Y$ is not an integer homology three-sphere, the absolute grading can be defined only for the summands of the Floer homology which belong to $\operatorname{Spin}^{c}$ structures over $Y$ whose first Chern class is torsion. Moreover, the absolute grading is no longer a $\mathbb{Z}$-grading in general, but rather, it gives a grading by rational numbers [22]. We do not discuss this in great generality, but rather content ourselves with the situation of three-manifolds $Y_{0}$ with first homology isomorphic to $\mathbb{Z}$.

In this case, let $H F^{+}\left(Y_{0}, 0\right) \subset H F^{+}\left(Y_{0}\right)$ denote the summand corresponding to the $\operatorname{Spin}^{c}$ structure with vanishing first Chern class. In this case, the absolute grading takes its values in rational numbers of the form $\frac{1}{2}+\mathbb{Z}$. The relationship between this absolute grading and the $\mathbb{Z} / 2 \mathbb{Z}$-grading referred to earlier is given by the relation that elements whose absolute $\mathbb{Q}$-grading lies in $-\frac{1}{2}+2 \mathbb{Z}$ have even parity, while those whose absolute $\mathbb{Q}$-grading lies in $\frac{1}{2}+2 \mathbb{Z}$ have odd parity.

For such three-manifolds, $H F^{\infty}$ is determined by [18, Theorem 10.1] to have the form

$$
H F^{\infty}\left(Y_{0}, 0\right) \cong \mathbb{Q}\left[U, U^{-1}\right] \oplus \mathbb{Q}\left[U, U^{-1}\right],
$$

where here the two summands have different parity. Correspondingly, there are now two numerical invariants analogous to the earlier correction term, $d_{ \pm 1 / 2}\left(Y_{0}\right)$ - the maximal $\mathbb{Q}$-grading of any element in $\mathrm{HF}^{+}\left(Y_{0}\right)$ contained in the image of $H F^{\infty}\left(Y_{0}\right)$ whose parity is given by $\pm \frac{1}{2}+2 \mathbb{Z}$.

These numerical invariants can be used to give a more precise form of Theorem 2.1 in the case where $i=0$. Specifically, we get the following straightforward combination of Theorem 10.17 with the discussion from [22, Section 4]:

$$
\chi\left(H F_{\text {red }}^{+}\left(S_{0}^{3}(K), 0\right)\right)-\left(\frac{d_{-1 / 2}\left(S_{0}^{3}(K)\right)-d_{1 / 2}\left(S_{0}^{3}(K)\right)+1}{2}\right)=-t_{0}(K)
$$

Note that it follows from the algebraic structure of $H F^{\infty}$ (specifically, an action of $H_{1}\left(Y_{0} ; \mathbb{Z}\right)$ on $\left.H F^{\infty}\left(Y_{0} ; \mathbb{Z}\right)\right)$ that

$$
d_{1 / 2}\left(Y_{0}\right)-1 \leq d_{-1 / 2}\left(Y_{0}\right)
$$

(cf [22, Proposition 4.10]). 


\subsection{More on twisted coefficients}

Throughout this section, $Y_{0}$ will denote a three-manifold with $H_{1}\left(Y_{0} ; \mathbb{Z}\right) \cong \mathbb{Z}$, and indeed we implicitly fix such an identification. This at once gives rise to an isomorphism $\operatorname{Spin}^{c}\left(Y_{0}\right) \cong \mathbb{Z}$ (via the map $\mathfrak{s} \mapsto c_{1}(\mathfrak{s}) / 2$, followed by the identification $H^{2}\left(Y_{0} ; \mathbb{Z}\right) \cong \mathbb{Z}$ induced by Poincaré duality), and we write $H F^{+}\left(Y_{0}, i\right)$ for the summand of $H_{F}^{+}\left(Y_{0}\right)$ in the $\operatorname{Spin}^{c}$ structure corresponding to the integer $i$. Similarly, there is an induced surjective homomorphism $H^{1}\left(Y_{0} ; \mathbb{Z}\right) \longrightarrow \mathbb{Z} / p \mathbb{Z}$

The following fact will be useful in the applications of Section 4.

Proposition 2.5 The rank of $\underline{H F_{\text {red,ev }}^{+}}\left(Y_{0} ; \mathbb{Q}[\mathbb{Z} / p \mathbb{Z}]\right)$, and respectively that of $\underline{H F}_{\text {red,odd }}^{+}\left(Y_{0} ; \mathbb{Q}[\mathbb{Z} / p \mathbb{Z}]\right)$, is a non-decreasing function of $p$. In fact, there is there is a short exact sequence

$$
0 \longrightarrow C \longrightarrow \underline{H F}_{\mathrm{red}}^{+}\left(Y_{0}\right) \otimes_{\mathbb{Q}\left[T, T^{-1}\right]} \mathbb{Q}[\mathbb{Z} / p \mathbb{Z}] \longrightarrow \underline{H F}_{\mathrm{red}}^{+}\left(Y_{0} ; \mathbb{Q}[\mathbb{Z} / p \mathbb{Z}]\right) \longrightarrow 0
$$

where $C$ is a $\mathbb{Z} / 2 \mathbb{Z}$-graded group of rank $\left(d_{-1 / 2}-d_{1 / 2}+1\right) / 2$ supported entirely in odd parity.

Before giving the proof, we prove another useful lemma (which ensures that the $\mathbb{Q}$-rank of the vector space in the middle of Equation (12) grows linearly with $p)$. Note $\underline{H F}+\left(Y_{0} ; \mathbb{Q}\left[T, T^{-1}\right]\right)$ is a direct sum of cyclic $\mathbb{Q}\left[T, T^{-1}\right]-$ modules. As such, it has a canonical torsion submodule whose quotient is a free $\mathbb{Q}\left[T, T^{-1}\right]$ module.

Lemma 2.6 The torsion submodule of $\underline{H F}^{+}\left(Y_{0} ; \mathbb{Q}\left[T, T^{-1}\right]\right)$ is in fact annihilated by $1-T$. Indeed, this torsion submodule is identified with the image of $\underline{H F^{\infty}}\left(Y_{0} ; \mathbb{Q}\left[T, T^{-1}\right]\right)$ in $\underline{H F}^{+}\left(Y_{0} ; \mathbb{Q}\left[T, T^{-1}\right]\right)$, and hence its quotient $\underline{H F}_{\mathrm{red}}^{+}\left(Y_{0} ; \mathbb{Q}\left[T, T^{-1}\right]\right)$ is a free $\mathbb{Q}\left[T, T^{-1}\right]$-module.

Proof This can be seen, for example, from the twisted long exact sequence. First, note that we can realize $Y_{0}$ as 0 -framed surgery along a knot $K$ in an integer homology three-sphere $Y$. This gives rise to an integral surgeries long exact sequence with twisted coefficients [18, Theorem 9.23], generalizing Theorem 2.2. In particular, when $p$ is sufficiently large, we get an exact sequence

$$
\begin{aligned}
\ldots \stackrel{\underline{a}}{\rightarrow} \underline{H F^{+}}\left(Y_{0}, i ; \mathbb{Q}[\mathbb{Z} / p \mathbb{Z}]\right) \stackrel{\underline{b}}{\rightarrow} H F^{+}\left(Y_{p},[i]\right) \otimes_{\mathbb{Q}} \mathbb{Q}[\mathbb{Z} / p \mathbb{Z}] \\
\stackrel{\underline{c}}{\longrightarrow} H F^{+}(Y) \otimes_{\mathbb{Q}} \mathbb{Q}[\mathbb{Z} / p \mathbb{Z}] \longrightarrow \ldots
\end{aligned}
$$


Note that [18, Theorem 9.23] is stated for the zero-surgery in its "universally twisted" case, i.e. with coefficients in $\mathbb{Z}[\mathbb{Z}]$, but this can be readily specialized to $\mathbb{Q}[\mathbb{Z} / p \mathbb{Z}]$ as above; also, in general, the group corresponding to the zerosurgery consists of a direct sum of groups $\underline{H F}+\left(Y_{0}, j ; \mathbb{Q}[\mathbb{Z} / p \mathbb{Z}]\right)$ over all $j \equiv i$ $(\bmod p)$, but here we apply this in the case where $p$ is sufficiently large that each of these terms has only one non-zero summand.

The image of $\underline{a}$ is easily seen to coincide with the image of $\underline{H F}{ }^{\infty}\left(Y_{0} ; \mathbb{Q}[\mathbb{Z} / p \mathbb{Z}]\right)$ inside $\underline{H F}+\left(Y_{0} ; \mathbb{Q}[\mathbb{Z} / p \mathbb{Z}]\right)$; while the image of $\underline{b}$ is a free $\mathbb{Q}[\mathbb{Z} / p \mathbb{Z}]$-module (since it is a submodule of a free $\mathbb{Q}[\mathbb{Z} / p \mathbb{Z}]$-module).

According to [18, Theorem 10.12],

$$
\underline{H F}^{\infty}\left(Y_{0}, 0 ; \mathbb{Q}[\mathbb{Z}]\right) \cong \mathbb{Q}\left[U, U^{-1}\right],
$$

thought of as a $\mathbb{Q}[\mathbb{Z}]$-module with trivial action by $\mathbb{Z}$. (Indeed, a result of this type holds for all three-manifolds and this is what is used to define the canonical $\mathbb{Z} / 2 \mathbb{Z}$-grading in general.) From the universal coefficients theorem, it follows readily now that

$$
\underline{H F}^{\infty}\left(Y_{0}, 0 ; \mathbb{Q}[\mathbb{Z} / p \mathbb{Z}]\right) \cong \mathbb{Q}\left[U, U^{-1}\right] \oplus \mathbb{Q}\left[U, U^{-1}\right]
$$

for all $p>0$, where here the two summands have different parity. It might appear that this gives a new pair of correction terms $d_{ \pm 1 / 2}\left(Y_{0} ; \mathbb{Q}[\mathbb{Z} / p \mathbb{Z}]\right)$. However, we have the following:

Lemma 2.7 The correction term $d_{ \pm 1 / 2}(Y ; \mathbb{Q}[\mathbb{Z} / p \mathbb{Z}])$ is independent of $p$ (ie it agrees with the correction term $\left.d_{ \pm 1 / 2}(Y)\right)$.

Proof We have $\mathbb{Q}\left[T, T^{-1}\right]-$ module maps

$$
\mathbb{Q} \stackrel{\eta}{\longrightarrow} \mathbb{Q}[\mathbb{Z} / p \mathbb{Z}] \stackrel{\epsilon}{\longrightarrow} \mathbb{Q}
$$

where $\eta(1)=\sum_{i=0}^{p-1} T^{i}$ and $\epsilon\left(T^{i}\right)=1$, so that $\epsilon \circ \eta$ is multiplication by $p$ (ie an isomorphism). In the notation, we use here an identification $\mathbb{Q}[\mathbb{Z} / p \mathbb{Z}] \cong$ $\mathbb{Q}[T] / T^{p}-1$. These homomorphisms induce maps on the Floer homologies with twisted coefficients; and indeed we get a diagram:

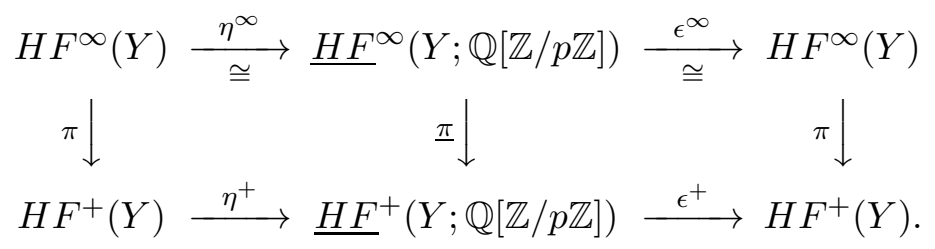


On the chain level, $\epsilon^{+} \circ \eta^{+}$is multiplication by $p$, and hence the induced maps on homology compose to give an isomorphism. Indeed, by the universal coefficients theorem, both $\eta^{\infty}$ and $\epsilon^{\infty}$ induce isomorphisms. The statement about the correction terms now follows from a diagram chase of Equation (14).

Now, we prove Proposition 2.5

Proof of Proposition 2.5 The universal coefficients theorem gives a diagram

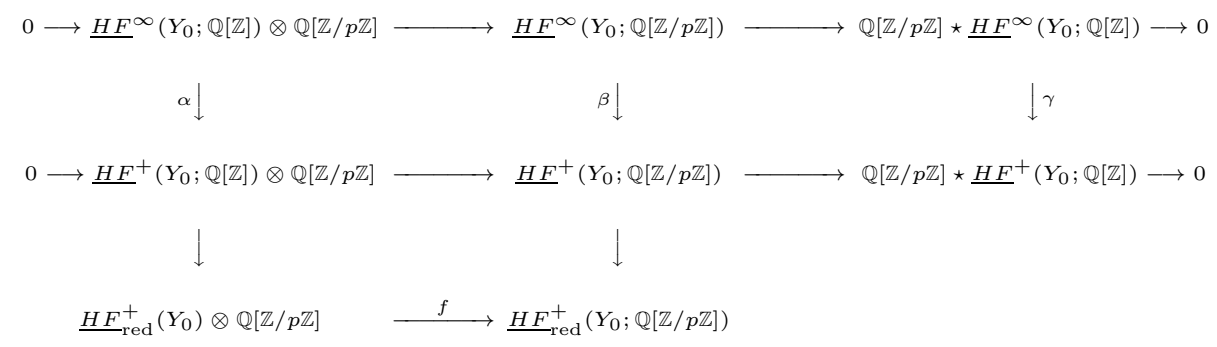

We claim first that $f$ is surjective. This follows from the fact that $\gamma$ is surjective, a fact which we establish using the following diagram



since according to Lemma 2.6 the kernel of $1-T$ (on the bottom row) is contained in the image of $\underline{\pi}$.

Now, the kernels of $\alpha, \beta$, and $\gamma$ and the maps between them are independent of $p$ : they are determined by $d_{ \pm 1 / 2}\left(Y_{0} ; \mathbb{Q}[\mathbb{Z} / p \mathbb{Z}]\right)$, which we saw in Lemma 2.7 to be independent of $p$. In particular, the cokernel of the induced map from $\operatorname{ker} \beta$ to $\operatorname{ker} \gamma$ (which in turn is identified with the kernel of $f$ ) is a group supported entirely in odd parity, whose rank is independent of $f$. Indeed, it is not hard to see that this rank is given by $\left(d_{-1 / 2}\left(Y_{0}\right)-d_{1 / 2}\left(Y_{0}\right)+1\right) / 2$. This provides us with the claimed exact sequence from equation (12), establishing the proposition.

It is worth noting the following quick consequence of the above proposition:

$$
\begin{aligned}
& \chi\left(\underline{H F}_{\mathrm{red}}^{+}\left(Y_{0}, 0 ; \mathbb{Q}[\mathbb{Z} / p \mathbb{Z}]\right)\right) \\
& \quad-\left(\frac{d_{-1 / 2}\left(Y_{0} ; \mathbb{Q}[\mathbb{Z} / p \mathbb{Z}]\right)-d_{1 / 2}\left(Y_{0} ; \mathbb{Q}[\mathbb{Z} / p \mathbb{Z}]\right)+1}{2}\right)=-p \cdot t_{0}(K) .
\end{aligned}
$$




\section{Relationship with Heegaard Floer homology for the zero-surgery}

Let $Y$ be a Seifert fibered space with $b_{1}(Y)=0$ or 1 . Such a manifold can be realized as the boundary of a four-manifold $W(\Gamma)$ obtained by plumbing two-spheres according to a weighted tree $\Gamma$. Here, the weights are thought of as a map $m$ from the set of vertices of $\Gamma$ to $\mathbb{Z}$.

Definition 3.1 Suppose that $\Gamma$ is a weighted tree which has either negativedefinite or negative-semi-definite intersection form. Then, we say that the induced orientation $-\partial W(\Gamma)$ is a positive Seifert orientation.

Note that if $Y$ is a Seifert fibered space with $b_{1}(Y)=0$ then at least one of $+Y$ or $-Y$ has a positive Seifert orientation. Moreover, either orientation on any lens space is a positive Seifert orientation; similarly, either orientation on a Seifert fibered space with $b_{1}(Y)=1$ is a positive Seifert orientation. Finally, if $Y$ is the quotient of a circle bundle $\pi: N \longrightarrow \Sigma$ over a Riemann surface by a finite group of orientation-preserving automorphisms $G$, and if $N$ is oriented as a circle bundle with positive degree, then the induced orientation on $Y$ is a positive Seifert orientation. An integral homology Seifert fibered space $Y$ has positive Seifert orientation if and only if its Casson invariant is positive, cf [4].

Following [25], we work with the following convenient generalization of the notion of positively oriented Seifert fibered spaces:

Definition 3.2 Let $\Gamma$ be a weighted graph which is a disjoint union of trees. The degree of a vertex $d(v)$ is the number of edges which contain $v$. We say that $\Gamma$ is a negative-definite (respectively negative-semi-definite) graph with at most one bad point if the intersection form for $\Gamma$ is negative-definite (respectively negative-semi-definite), and there is at most one vertex $v \in \Gamma$ whose weight $m(v)$ is larger than $-d(v)$.

For three-manifolds $Y=-\partial W(\Gamma))$, where $\Gamma$ is a negative-definite graph with at most one bad point, $H F^{+}(Y)$ can be explitly calculated in terms of the graph $\Gamma$, see [25]. Indeed, the part of that calculation which we will use in the present paper can be summarized as follows:

Theorem 3.3 Let $\Gamma$ be a negative-definite graph with at most one bad point. Then, $H F^{+}(-\partial W(\Gamma))$ is supported in even dimensions. Moreover, if $\Gamma$ has no bad points, then $H F_{\text {red }}^{+}(-\partial W(\Gamma))=0$. 
Proof These statements are Corollary 1.4 and Lemma 2.6 respectively from [25].

We state the following result in the case of either integral surgeries or $1 / q$ surgeries for integral $q$. However, a corresponding statement can also be proved for $p / q$ surgeries by adapting arguments from [13, where an analogous result is proved using the Seiberg-Witten monopole equations. We do not pursue this generalization here, however.

Theorem 3.4 Let $K \subset S^{3}$ be a knot in the three-sphere, and suppose that there is an integer $p \geq 0$ and a negative definite or semi-definite graph $\Gamma$ with only one bad point with the property that

$$
S_{p}^{3}(K) \cong-\partial W(\Gamma)
$$

then all the elements of $H F_{\text {red }}^{+}\left(S_{0}^{3}(K)\right)$ have odd $\mathbb{Z} / 2 \mathbb{Z}$-grading. Similarly, if $p>0$ and

$$
S_{1 / p}^{3}(K) \cong-\partial W(\Gamma),
$$

all the elements of a twisted Floer homology group $\underline{H F_{\text {red }}^{+}}\left(S_{0}^{3}(K), \mathbb{Q}[\mathbb{Z} / p \mathbb{Z}]\right)$ have odd $\mathbb{Z} / 2 \mathbb{Z}$-grading. In particular, in either case, all the torsion coefficients $t_{i}(K)$ (cf equation (10) are non-negative.

Proof If $\Gamma$ is a weighted graph, we let $Y(\Gamma)$ denote the oriented threemanifold obtained as $\partial W(\Gamma)$.

Assume first that $p \neq 0$, and $S_{p}^{3}(K) \cong-Y(\Gamma)$. Consider now the integer surgeries long exact sequence, Theorem 2.2 (cf [18, Theorem 9.19]), according to which for each $i \in \mathbb{Z} / p \mathbb{Z}$, we have the long exact sequence

$$
\begin{aligned}
& \ldots \stackrel{c}{\longrightarrow} H F^{+}\left(S^{3}\right) \stackrel{a}{\longrightarrow} \bigoplus_{\{j \in \mathbb{Z} \mid j \equiv i(\bmod p)\}} H F^{+}\left(S_{0}^{3}(K), j\right) \\
& \stackrel{b}{\longrightarrow} H F^{+}\left(S_{p}^{3}(K), i\right) \stackrel{c}{\longrightarrow} \ldots
\end{aligned}
$$

where the maps $a$ and $c$ preserve the absolute $\mathbb{Z} / 2 \mathbb{Z}$-grading, and $b$ reverses it. We assume that $S_{p}^{3}(K) \cong-Y(\Gamma)$. Consider an element $\xi \in H F^{+}\left(S_{0}^{3}(K)\right)$ whose absolute $\mathbb{Z} / 2 \mathbb{Z}$-grading is even. Then it follows from Theorem 3.3 that $b(\xi)=0$, hence that $\xi \in \operatorname{Im} a$, from which it follows at once that $\xi$ comes from $H F^{\infty}\left(S_{0}^{3}(K)\right)$, in particular, it maps trivially to $H F_{\text {red }}^{+}\left(S_{0}^{3}(K)\right)=$ $H F^{+}\left(S_{0}^{3}(K)\right) / H F^{\infty}\left(S_{0}^{3}(K)\right)$. Thus, $H F_{\text {red }}^{+}\left(S_{0}^{3}(K)\right)$ is supported in odd grading, as claimed. 
For $1 / p$-surgeries with $p \neq 0$, we can repeat the above argument, only now using the fractional surgeries long exact sequence, Theorem 2.3] (cf [18, Theorem 9.14]), which now reads

$$
\ldots \stackrel{c}{\longrightarrow} H F^{+}\left(S^{3}\right) \stackrel{a}{\longrightarrow} \underline{H F^{+}}\left(S_{0}^{3}(K), \mathbb{Q}[\mathbb{Z} / p \mathbb{Z}]\right) \stackrel{b}{\longrightarrow} H F^{+}(-Y(\Gamma)) \stackrel{c}{\longrightarrow} \ldots
$$

For the statement of the result where $p=0$, we extend the methods of 25 , Section 2] to prove that for negative semi-definite graphs $\Gamma$ with at most one bad point, $H F_{\text {red }}^{+}(-Y(\Gamma))$ is supported in odd degrees. To this end, if $v$ is a vertex in a marked graph $\Gamma$, we let $\Gamma_{-1}(v)$ denote the marked graph which agrees with $\Gamma$, except that the weight of $v$ for $\Gamma_{-1}(v)$ is one less than the weight of $v$ for $\Gamma$. Now, the surgery long exact sequence, in the form it is used in 25 , Proposition 2.8], gives:

$$
\ldots \stackrel{c}{\longrightarrow} H F^{+}(-Y(\Gamma-v)) \stackrel{a}{\longrightarrow} H F^{+}(-Y(\Gamma)) \stackrel{b}{\longrightarrow} H F^{+}\left(-Y\left(\Gamma_{-1}(v)\right) \stackrel{c}{\longrightarrow} \ldots\right.
$$

and again, $a$ and $c$ preserve $\mathbb{Z} / 2 \mathbb{Z}$-grading while $b$ reverses it. It is straightforward to see now that $\Gamma_{-1}(v)$ is a negative-definite graph with at most one bad point, and hence Theorem 3.3 applies to it. Thus, if $\xi \in H F_{\mathrm{ev}}^{+}\left(S_{0}^{3}(K)\right)$, then its image under $b$ is trivial, and hence $\xi=a(\eta) \in H F^{+}(-Y(\Gamma-v))$. Indeed, since $\Gamma-v$ has no bad points, Theorem 3.3 ensures that $H F_{\text {red }}^{+}(-Y(\Gamma-v))=0$, ie $\eta$ comes from $H F^{\infty}(-Y(\Gamma-v))$, and hence $\xi$ comes from $H F^{\infty}(-Y(\Gamma))$, proving the claim.

For the statement about the Alexander polynomial we appeal to Theorem 2.1 according to which when $i \neq 0$,

$$
\chi\left(H F^{+}\left(S_{0}^{3}(K), i\right)\right)=-t_{i}(K)
$$

(in this case $\left.H F^{+}\left(S_{0}^{3}(K), i\right)=H F_{\text {red }}^{+}\left(S_{0}^{3}(K), i\right)\right)$; while in the case where $i=0$, we still have that

$$
\chi\left(H F_{\text {red }}^{+}\left(S_{0}^{3}(K), 0\right)\right) \geq-t_{0}(K) .
$$

For the fractional surgeries case, we use the fact that

$$
\chi\left(\underline{H F}^{+}\left(Y_{0}, i, \mathbb{Q}[\mathbb{Z} / p \mathbb{Z}]\right)\right)=-p \cdot t_{i}(K)
$$

when $i \neq 0$ (this is a combination of equation (9) and Theorem 2.11), and

$$
\chi\left(\underline{H F}_{\mathrm{red}}^{+}\left(S_{0}^{3}(K), 0, \mathbb{Q}[\mathbb{Z} / p \mathbb{Z}]\right)\right) \geq-p \cdot t_{0}(K)
$$

(this follows from equation (15), together with inequality (11)).

In the case of negative Seifert orientation, we have the following: 
Proposition 3.5 Let $q>0$ be an integer, and suppose that $S_{1 / q}^{3}(K)$ is a Seifert fibered space with negative Seifert orientation. Then all the elements of $\underline{H F_{\text {red }}^{+}}\left(S_{0}^{3}(K), \mathbb{Q}[\mathbb{Z} / q \mathbb{Z}]\right)$ have even $\mathbb{Z} / 2 \mathbb{Z}$-grading. Moreover,

$$
d_{1 / 2}\left(S_{0}^{3}(K)\right)=\frac{1}{2} \quad \text { and } \quad d_{-1 / 2}\left(S_{0}^{3}(K)\right)=-\frac{1}{2} .
$$

Proof Consider first for notational simplicity the case where $q=1$.

According to [18, Proposition 2.5], if $Y$ is any rational homology three-sphere, then $H F_{\text {red }}^{+}(Y)$

$\cong H F_{\text {red }}^{+}(-Y)$, under a map which reverses the $\mathbb{Z} / 2 \mathbb{Z}$-grading. Combining Theorem 3.3 with the surgery long exact sequence we see that $H F_{\text {red,ev }}^{+}\left(S_{1}^{3}(K)\right)=0$. It follows in turn from this together with the surgery long exact sequence that $H F^{+}\left(S^{3}\right)$ injects into $\mathrm{HF}^{+}\left(S_{0}^{3}(K)\right)$ and hence again by the exact sequence that the natural map from $H F_{\text {odd }}^{+}\left(S_{0}^{3}(K)\right)$ to $H F_{\mathrm{ev}}^{+}\left(S_{1}^{3}(K)\right)$ induces an isomorphism and in particular that $H F_{\text {red,odd }}^{+}\left(S_{0}^{3}(K)\right)=0$.

For all $i \neq 0, H F_{\text {red }}^{+}\left(S_{0}^{3}(K), i\right)=H F^{+}\left(S_{0}^{3}(K)\right)$. Thus, we can conclude from Theorem 2.1 that for all $i \neq 0, t_{i}(K)<0$.

Indeed, the injectivity of $H F^{+}\left(S^{3}\right)$ in $H F^{+}\left(S_{0}^{3}(K)\right)$ ensures $d_{-1 / 2}\left(S_{0}^{3}(K)\right)=$ $-1 / 2$. This implies that

$$
d_{1 / 2}\left(S_{0}^{3}(K)\right) \leq 1 / 2,
$$

according to inequality (11). Now, since the map from $H F_{\text {odd }}^{+}\left(S_{0}^{3}(K), 0\right)$ to $H F_{\text {ev }}^{+}\left(S_{1}^{3}(K)\right)$ drops degree by $1 / 2$ we see that $d\left(S_{1}^{3}(K)\right) \leq d_{1 / 2}\left(S_{0}^{3}(K)\right)-1 / 2$. But a negatively oriented Seifert fibered space bounds a smooth, negativedefinite four-manifold (given by its plumbing description), so we can apply Theorem 2.4 to conclude that $d_{1 / 2}\left(S_{0}^{3}(K)\right) \geq 1 / 2$. Putting this together with equation (16), we conclude that $d_{1 / 2}\left(S_{0}^{3}(K)\right)=1 / 2$.

In the case where $q>1$, we use the long exact sequence for fractional surgeries (Theorem 2.3) to obtain the corresponding statement for twisted coefficients. Note that the correction terms are independent of the choice of $q$, according to Lemma 2.7

Proof of Theorem 1.1 Assume $S_{1 / q}^{3}(K)$ is Seifert fibered. By reflecting $K$ if necessary, we can assume that $q>0$.

When $S_{1 / q}^{3}(K)$ is a positively oriented Seifert space, Theorem 3.4 shows that $\underline{H F_{\text {red }}^{+}}\left(S_{0}^{3}(K)\right)$ has odd parity. We then use Theorem 2.1 to conclude that all the torsion coefficients of $K$ are non-negative (equation (9) for $i \neq 0$ and equation 
(15) for $i=0)$. In the case where $S_{1 / q}^{3}(K)$ is a negatively oriented Seifert fibered space, we use Proposition [3.5, to conclude that $\underline{H F_{\text {red }}^{+}}\left(S_{0}^{3}(K), \mathbb{Q}[\mathbb{Z} / p \mathbb{Z}]\right)$ is supported in even parity. From Proposition 2.5 we conclude that $\underline{H F}_{\mathrm{red}}^{+}\left(S_{0}^{3}(K)\right)$ is supported in even parity as well. The Euler characteristic relations (Theorem 2.1. together with equations (9) and (15) now imply that all the torsion coefficients are non-positive in this case.

\section{Relationship with the knot Floer homology}

Theorem 1.4 follows from Theorem 3.4 together with the relationship between $\widehat{H F K}\left(S^{3}, K\right)$ and the Heegaard Floer homology of surgeries along $K$ developed in [21, Section 4], see also [28].

In [21, Corollary 4.5], it is shown that if $\widehat{H F K}\left(S^{3}, K, i\right)=0$ for all $i>d$, then $\widehat{H F K}\left(S^{3}, K, d\right) \cong H F^{+}\left(S_{0}^{3}(K), d-1\right)$ as relatively $\mathbb{Z} / 2 \mathbb{Z}$-graded Abelian groups. (With the conventions of $[18$, the isomorphism here reverses the $\mathbb{Z} / 2 \mathbb{Z}-$ grading.) In the following lemma, we give the relevant statement when $d=1$.

Lemma 4.1 If $\widehat{H F K}\left(S^{3}, K, i\right)=0$ for all $|i|>1$, then

$$
H F_{\text {red,ev }}^{+}\left(S_{0}^{3}(K), 0\right) \cong \widehat{H F K}_{\text {odd }}\left(S^{3}, K, 1\right) .
$$

Proof We use [21, Theorem 4.4]. According to that theorem, we have a $\mathbb{Z} \oplus \mathbb{Z}-$ filtered complex $C=C F K^{\infty}\left(S^{3}, K, 0\right)$, which admits quotient complexes

$$
\begin{aligned}
C\{i \geq 0 \text { or } j \geq d-1\} & \sim C F^{+}\left(S_{p}^{3}(K),[d-1]\right), \\
C\{i \geq 0\} & \sim C F^{+}\left(S^{3}\right)
\end{aligned}
$$

where here $\sim$ denotes relatively graded, absolutely $\mathbb{Z} / 2 \mathbb{Z}$-graded chain homotopy equivalence, $C\{i \geq 0, j \geq 0\}$ denotes the quotient complex of $C$ by all elements whose filtration level $(i, j)$ has both $i<0$ and $j<0, p$ is any sufficiently large positive integer, and $[d-1]$ is a $\operatorname{Spin}^{c}$ structure over $S_{p}^{3}(K)$, which is naturally $\operatorname{Spin}^{c}$-cobordant to a $\operatorname{Spin}^{c}$ structure over $S_{0}^{3}(K)$ whose first Chern class is $2(d-1)$ times a generator of $H^{2}\left(S_{0}^{3}(K) ; \mathbb{Z}\right)$. For more on this, see [21, Section 4]. Clearly, we have the short exact sequence

$$
0 \longrightarrow C\{i<0 \text { and } j \geq d-1\} \longrightarrow C\{i \geq 0 \text { or } j \geq d-1\} \longrightarrow C\{i \geq 0\} \longrightarrow 0
$$

The hypothesis that $\widehat{H F K}\left(S^{3}, K, j\right)=0$ for all $j>d$ ensures, by taking filtrations, that

$$
H_{*}(C\{i<0 \text { and } j \geq d-1\}) \cong \widehat{H F K}\left(S^{3}, K, d\right) .
$$


Thus, we obtain the long exact sequence

$$
\ldots \longrightarrow \widehat{H F K}\left(S^{3}, K, d\right) \longrightarrow H F^{+}\left(S_{p}^{3}(K),[d-1]\right) \longrightarrow H F^{+}\left(S^{3}\right) \stackrel{\delta}{\longrightarrow} \ldots
$$

As the above maps are $U$-equivariant, it is easy to see (from the $\mathbb{Z}[U]$-module structure of $\left.H F^{+}\left(S^{3}\right)\right)$ that $\delta$ is the trivial map, thus $H F^{+}\left(S_{p}^{3}(K),[d-1]\right)$ contains a $\mathbb{Z}[U]$-submodule isomorphic to $\widehat{H F K}\left(S^{3}, K, d\right)$. In fact, we have that

$$
\widehat{H F K}_{\text {odd }}\left(S^{3}, K, d\right) \cong H F_{\text {odd }}^{+}\left(S_{p}^{3}(K),[d-1]\right) .
$$

Specializing to the case where $d=1$, we consider the integral surgeries long exact sequence. This gives exactness for

$$
H F_{\mathrm{ev}}^{+}\left(S^{3}\right) \longrightarrow H F_{\mathrm{ev}}^{+}\left(S_{0}^{3}(K), 0\right) \longrightarrow H F_{\text {odd }}^{+}\left(S_{p}^{3}(K),[0]\right) \longrightarrow 0 .
$$

It is easy to see that the image of $H F_{\mathrm{ev}}^{+}\left(S^{3}\right)$ inside $H F_{\mathrm{ev}}^{+}\left(S_{0}^{3}(K), 0\right)$ coincides with the image of $H F_{\mathrm{ev}}^{\infty}\left(S_{0}^{3}(K), 0\right)$ inside $H F_{\mathrm{ev}}^{+}\left(S_{0}^{3}(K), 0\right)$. Thus,

$$
H F_{\text {red,ev }}^{+}\left(S_{0}^{3}(K), 0\right) \cong H F_{\text {red,odd }}^{+}\left(S_{p}^{3}(K),[0]\right)=H F_{\text {odd }}^{+}\left(S_{p}^{3}(K),[0]\right)
$$

Proof of Theorem 1.4. According to [21, Corollary 4.5], if $\widehat{H F K}\left(S^{3}, K, i\right)=$ 0 for all $i>d>1$, then

$$
\widehat{H F K}\left(S^{3}, K, d\right) \cong H F^{+}\left(S_{0}^{3}(K), d-1\right)
$$

under an isomorphism which reverses parity.

Suppose that $S_{1 / p}^{3}(K)$ is a positively oriented Seifert fibered space. According to Theorem [3.4 $\underline{H F}_{\text {red }}^{+}\left(S_{0}^{3}(K) ; \mathbb{Q}[\mathbb{Z} / p \mathbb{Z}]\right)$ is supported in odd degrees. From this and Proposition [2.5] we can conclude the same for $H F_{\text {red }}^{+}\left(S_{0}^{3}(K)\right)$. When the genus of $K$ is greater than one, we have that

$$
H F^{+}\left(S_{0}^{3}(K), g-1\right)=H F_{\text {red }}^{+}\left(S_{0}^{3}(K), g-1\right),
$$

and hence all its elements have odd parity. Thus, in view of equation (17), $\widehat{H F K}\left(S^{3}, K, g\right)$ is supported in even degrees (and it is non-trivial, cf equation (5)). The case where $g=1$ follows from a similar argument, using Lemma 4.1 in place of equation (17)

Suppose that $g>1$ and $S_{1 / p}^{3}(K)$ is a negatively oriented Seifert fibered space. Now, according to Proposition $\left[3.5\right.$ we conclude that $\underline{H F_{\text {red }}^{+}}\left(S_{0}^{3}(K) ; \mathbb{Q}[\mathbb{Z} / p \mathbb{Z}]\right)$ is supported entirely in even degrees, and hence according to Proposition 2.5 it follows that $H F_{\text {red }}^{+}\left(S_{0}^{3}(K)\right)$ is supported entirely in even degrees. Since $g>1$, the same can be said about $H^{+}\left(S_{0}^{3}(K), g-1\right)$. From equation (17) it now follows that $\widehat{H F K}(K, g)$ is supported in odd degrees (and it is again non-trivial according to equation (5) ). 


\section{$5 \quad$ Surgeries giving $\Sigma(2,3,5)$ and $\Sigma(2,3,7)$}

In general, if $Y$ is a three-manifold and $K \subset S^{3}$ is a knot with the property that $S_{r}^{3}(K) \cong Y$, then the exact sequences determine the Floer homology of the zero-surgery only up to some indeterminacy. However, in cases where the Floer homology of $Y$ has sufficiently small rank, this indeterminacy is eliminated.

Proof of Theorem 1.6 From Casson's invariant, it follows that if $S_{1 / q}^{3}(K)=$ $\Sigma(2,3,5)$, then $q= \pm 1$. Indeed, by reversing orientation if necessary, we have a knot with $S_{ \pm 1}^{3}(K)=-\Sigma(2,3,5)$. It follows from Donaldson's diagonalization theorem that the surgery coefficient must be +1 . Next, we apply the long exact sequence for +1 surgeries, together with the fact that $H^{+}(-\Sigma(2,3,5)) \cong \mathcal{T}^{+}$, with $d(\Sigma(2,3,5))=-2$. It follows easily that $\mathrm{HF}^{+}\left(S_{0}^{3}(K)\right)$ is uniquely determined from the fact that $S_{1}^{3}(K)=-\Sigma(2,3,5)$ (compare also 22, Proposition 8.1]). Indeed, we see that $H F^{+}\left(S_{0}^{3}(K)\right) \cong \mathcal{T}^{+} \oplus \mathcal{T}^{+}$, with $d_{-1 / 2}\left(S_{0}^{3}(K)\right)=$ $-1 / 2$ and $d_{1 / 2}\left(S_{0}^{3}(K)\right)=-3 / 2$. In particular, for all $i \neq 0, H F^{+}\left(S_{0}^{3}(K), i\right)=$ 0 . This establishes that the Floer homology of $S_{0}^{3}(K)$ is the same as the Floer homology of $S_{0}^{3}\left(T_{2,3}\right)$, where $T_{2,3}$ denotes the right-handed trefoil, and in particular, so is its Alexander polynomial. The fact that the knot Floer homology coincides with that of the trefoil follows from the main result of [17 (cf 17. Theorem 1.2]), according to which if $K \subset S^{3}$ satisfies the property that $H F_{\text {red }}^{+}\left(S_{p}^{3}(K)\right)=0$ for some integer $p$, then $\widehat{H F K}$ is uniquely determined by the Alexander polynomial of $K$. The remark about the Seifert genus follows from equation (5) ([26, Theorem 1.2]).

Proof of Theorem 1.7 Again, from Casson's invariant it follows at once that if $S_{r}^{3}(K)=\Sigma(2,3,7)$, then $r= \pm 1$. In the case where $S_{-1}^{3}(K) \cong \Sigma(2,3,7)$, a chase of the surgery long exact sequence shows that $H F^{+}\left(S_{0}^{3}(K)\right)$ coincides with that for the the right-handed trefoil; and in particular $\mathrm{HF}^{+}\left(S_{0}^{3}(K), i\right)=0$ for all $i \neq 0$. Now, by equation (17), it follows that $\widehat{H F K}\left(S^{3}, K, d\right)=0$ for all $d>1$, and hence, by equation (5), the genus of $K$ is one.

In the case where $S_{+1}^{3}(K) \cong \Sigma(2,3,7)$, a chase of the surgery long exact sequence once again yields that $\mathrm{HF}^{+}\left(S_{0}^{3}(K)\right)$ is uniquely determined, in particular, it is isomorphic to $\mathrm{HF}^{+}\left(S_{0}^{3}\left(K_{0}\right)\right)$, where here $K_{0}$ is the figure eight knot. More explicitly, $H F^{+}\left(S_{0}^{3}(K)\right)=\bigoplus_{i \in \mathbb{Z}} H F^{+}\left(S_{0}^{3}(K), i\right) \cong \mathcal{T}^{+} \oplus \mathcal{T}^{+} \oplus \mathbb{Q}$, (where the last summand has even parity) and $d_{ \pm 1 / 2}\left(S_{0}^{3}(K)\right)= \pm 1 / 2$. We complete the argument as before. 


\section{References}

[1] R Crowell, Genus of alternating link types, Ann. of Math. 69 (1959) 258-275 MR0099665

[2] J C Dean, Small Seifert fibered Dehn surgery on hyperbolic knots, Algebr. Geom. Topol. 3 (2003) 435-472 MR1997325

[3] M Eudave-Muñoz, On hyperbolic knots with Seifert fibered Dehn surgeries, from: "Proceedings of the First Joint Japan-Mexico Meeting in Topology (Morelia, 1999)". Topology Appl. 121 (2002) 119-141 MR1903687

[4] R Fintushel, R J Stern, Instanton homology of Seifert fibred homology threespheres, Proc. London Math. Soc. 61 (1990) 109-137 MR1051101

[5] K A Frøyshov, The Seiberg-Witten equations and four-manifolds with boundary, Math. Res. Lett. 3 (1996) 373-390 MR1397685

[6] K A Frøyshov, Equivariant aspects of Yang-Mills Floer theory, Topology 41 (2002) 525-552 MR1910040

[7] S Fukuhara, Y Matsumoto, K Sakamoto, Casson's invariant of Seifert homology 3-spheres, Math. Ann. 287 (1990) 275-285 MR1054569

[8] D Gabai, Genera of the arborescent links, Mem. Amer. Math. Soc. 59 (1986) i-viii, 1-98 MR0823442

[9] C McA Gordon, Dehn surgery on knots, from: "Proceedings of the International Congress of Mathematicians Vol. I (Kyoto, 1990)", Math. Soc. Japan, Tokyo (1991) 631-642 MR1159250

[10] M Gromov, Pseudoholomorphic curves in symplectic manifolds, Invent. Math. 82 (1985) 307-347 MR0809718

[11] S Kinoshita, H Terasaka, On unions of knots, Osaka Math. J. 9 (1957) 131153 MR0098386

[12] R Kirby, editor, Problems in low-dimensional topology, from: "Geometric topology (Athens, GA, 1993)", AMS/IP Stud. Adv. Math. 2, Amer. Math. Soc. Providence, RI (1997) 35-473 MR0098386

[13] P B Kronheimer, T S Mrowka, Z Szabó, P S Ozsváth, Monopoles and lens space surgeries, arXiv:math.GT/0310164

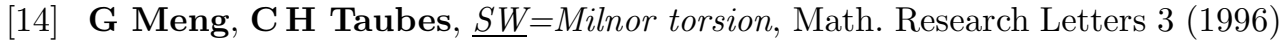
661-674 MR1418579

[15] K Murasugi, On the genus of the alternating knot I, II, J. Math. Soc. Japan, 10 (1958) 94-105, 235-248 MR0099664

[16] W Neumann, J Wahl, Casson invariant of links of singularities, Comment. Math. Helv. 65 (1990) 58-78 MR1036128

[17] P S Ozsváth, Z Szabó, On knot Floer homology and lens space surgeries, arXiv:math.GT/0303017, to appear in Topology 
[18] P S Ozsváth, Z Szabó, Holomorphic disks and three-manifold invariants: properties and applications, arXiv:math.SG/0105202 to appear in Ann. of Math.

[19] P S Ozsváth, Z Szabó, Holomorphic disks and topological invariants for closed three-manifolds, arXiv:math.SG/0101206 to appear in Ann. of Math.

[20] P S Ozsváth, Z Szabó, Holomorphic triangles and invariants for smooth fourmanifolds, arXiv:math.SG/0110169

[21] P S Ozsváth, Z Szabó, Holomorphic disks and knot invariants, Adv. Math. 186 (2004) 58-116 MR2065507

[22] P S Ozsváth, Z Szabó, Absolutely graded Floer homologies and intersection forms for four-manifolds with boundary, Adv. Math. 173 (2003) 179-261 MR1957829

[23] P S Ozsváth, Z Szabó, Heegaard Floer homology and alternating knots, Geom. Topol. 7 (2003) 225-254 MR1988285

[24] P S Ozsváth, Z Szabó, Knot Floer homology, genus bounds, and mutation, Topology Appl. 141 (2004) 59-85 MR2058681

[25] P S Ozsváth, Z Szabó, On the Floer homology of plumbed three-manifolds, Geom. Topol. 7 (2003) 185-224 MR1988284

[26] PS Ozsváth, Z Szabó, Holomorphic disks and genus bounds, Geom. Topol. 8 (2004) 311-334 MR2023281

[27] J A Rasmussen, Floer homology of surgeries on two-bridge knots, Algebr. Geom. Topol. 2 (2002) 757-789 MR1928176

[28] J A Rasmussen, Floer homology and knot complements, PhD thesis, Harvard University (2003), arXiv:math.GT/0306378

[29] V Turaev, A combinatorial formulation for the Seiberg-Witten invariants of 3-manifolds, Math. Res. Lett. 5 (1998) 583-598 MR1666856

[30] J R Weeks, SnapPea, http://www.geometrygames.org/weeks/

[31] X Zhang, On property I for knots in $S^{3}$, Trans. Amer. Math. Soc. 339 (1993) 643-657 MR1154545

Department of Mathematics, Columbia University, NY 10025, USA

Institute for Advanced Study, Princeton, NJ 08540, USA

and

Department of Mathematics, Princeton University, NJ 08544, USA

Email: petero@math.columbia.edu, szabo@math.princeton.edu

Received: 30 December $2003 \quad$ Revised: 28 April 2004 